\title{
INCLUSIVE CURRICULUM IN NEPAL'S CONTEXT
}

\author{
Lal-Shyãkarelu Rapacha, Ph.D., Chief Research Officer \\ National Foundation for Development of Indigenous Nationalities (NFDIN) \\ E-mail: kiranti.muru@hotmail.com
}

\begin{abstract}
Inclusive curriculum in contemporary Nepal's technical and higher education policy, programme, content and system is a dire need for making our education innovative technically and more competitive globally since our policy, programme, content and system till date have remained monolithic-thus feudalistic in structure as well as nature and in content mainly dominated by western colonial principle rather than by our own national indigenous knowledge. Nepal within her (its) national boundary, has a rich vein of indigenous knowledge (i.e. tangible and intangible cultural heritage ${ }^{1}$ including languages, cultures, archaeology, belief systems, ethnobotany, ethnomedicine etc) and skills (most of them are endangered and extinct now) just waiting to be tapped by the inclusive policy of government and private educational sector in the country. Therefore, the main purpose of this article is to explore new paradigm shift in our education policy, programme, content and system by making our curriculum much more inclusive in the process of knowledge-building and sustainability through an indigenous perspective with some empirical but selective and limited knowledge inventories.
\end{abstract}

KEYWORDS: Inclusive curriculum, indigenous peoples, knowledge inventories

\section{A GLIMPSE OF PAST AND PRESENT HISTORY AFFECTING EDUCATION}

Nepal's past history for millennia before the bloody rise and conquests of the House of Gorkha (cf. Pradhan xxiii) led by Prithivi Narayan Shah and the official imposition of Hinduism in 1854 (cf. Höfer 9-10 and Parker 145-165), was truly peaceful and prosperous linguistically, culturally, socioeconomically and truly autonomous federal politically. Utterly ruthless 'internal colonization' (cf. Leuthold 4) pushed the diverse-multifaceted assets and possibilities of the pluralistic nation into marginalization for ages by promoting homogenized (cf. Bayly 1-23) and monolithic policy of a single race, language and culture. Nepal now has shifted its political paradigm from monarchism to republicanism of late in 2006. Like in politics our education policy also now has to shit its paradigm by adopting inclusive curriculum with main focus to our own national indigenous knowledge and skills rather than western and national elite hegemony if we want independent viability and sustainability of our education programme, system, progress and development in every walk of our life.

\section{INCLUSIVE CURRICULUM}

In order to make Nepal's technical and higher education more innovative technically and more competitive globally through an indigenous perspective, there is no alternative for inclusive policy, programme and system of curriculum as it is a dire need of our contemporary time since the mission and principle of inclusion is guided by a commitment to equity, access and diversity. Nepal has a diverse demography of popula- 


\section{Crossing the Border: International Journal of Interdisciplinary Studies}

tion in relation to differences of languages, cultural background, gender, sexuality, age, family responsibility, religion and physical ability. This feature of the learning environment at our universities in Nepal is yet to be valued, articulated and negotiated in terms of the content and processes of the curriculum undoubtedly without delay.

\section{RATIONALES BEHIND INCLUSIVE CURRICULUM}

Nepal's universities till today hardly can fulfill students' expectations who come from diverse life experiences, linguistic and cultural backgrounds. Needless to say that good teaching acknowledges and values this diversity in effective curriculum planning. 'Inclusion' relates to increasing the participation and enhancing the quality of learning of all students vulnerable to exclusionary pressures. The term inclusive curriculum' includes the teaching and learning environment, the course content, the processes of teaching and learning, and the assessment practices. Good teaching includes the design of a curriculum that addresses all these features. Due to the lack of these inclusive features in our higher education policy, programme, content and curriculum, more than $90 \%$ of indigenous students are excluded from opportunity, capability, access and security.

An inclusive curriculum thus-

- recognizes that prior experiences inform students' expectations and experiences of the course,

- acknowledges and values the culture, language, background and experience of all students,

- is inclusive of gender, indigeneity/ethnicity, linguistic, cultural and socioeconomic background, age, sexuality, and differences related to ability and disability,

- is responsive and gives expression to the knowledge base of the students and staff in teaching and learning,

- acknowledges that any curriculum decision is a selection rather than a complete 'truth',

- makes explicit the rationales underpinning course design,

- makes clear the goals and standards, which include the key ideas or concepts of the discipline and the ways of arriving at an understanding of that discipline, and

- provides fair access to and distribution of resources.

(Source: cf. La Trobe University ${ }^{2}$ )

Our teachers till date from the inception of Tribhuvan University hardly bring a range of diversity issues to university (including technical training institutes/polytechnic) classroom teaching and learning contexts related to the students' own race, ethnicity, indigeneity, gender, age, linguistic and cultural background, physical attributes and abilities and their own attitudes towards diversity. They hardly realize that teachers' physical features and communication styles, and the extent to which these differ from the physical, cultural, linguistic and intellectual backgrounds of students, will influence the students' perceptions of the teaching and learning contexts and affect interactions in the classroom. Inclusive curriculum thus provides an equitable way to manage diversity is to create a safe atmosphere in which teachers' and the students' diversity can be included, discussed and valued. In this paper, now we shortly provide some glimpses of our diversities to be considered in the proposed inclusive curriculum. 


\section{INCLUSIVE CURRICULUM}

\section{INVENTORIES OF KNOWLEDGE/SKILLS}

Diverse indigenous populations, regions and biodiversity in Nepal (see Figure 1) have distinct nature and types of Aboriginal Indigenous Knowledge/Skills (AIK/S). Such AIK/Ss are recognized not only in Nepal but also have been recognized worldwide. It is not only because of its intrinsic value but also because it has a potential instrumental value to science and conservation (Kunwar and Bussmann 24). Here, the acronym AIK/S implies any knowledge or skill produced-by and or distinctive to a particular linguistic and cultural group or any loosely defined group of resource users in a given region (see Figure 1). Such knowledge or skill is usually produced informally by only people actually practicing a skill or working directly with are source (see Table 1), as opposed to paid knowledge professionals such as scientists.

Figure1: Nepal's diverse indigenous populations, regions and biodiversity

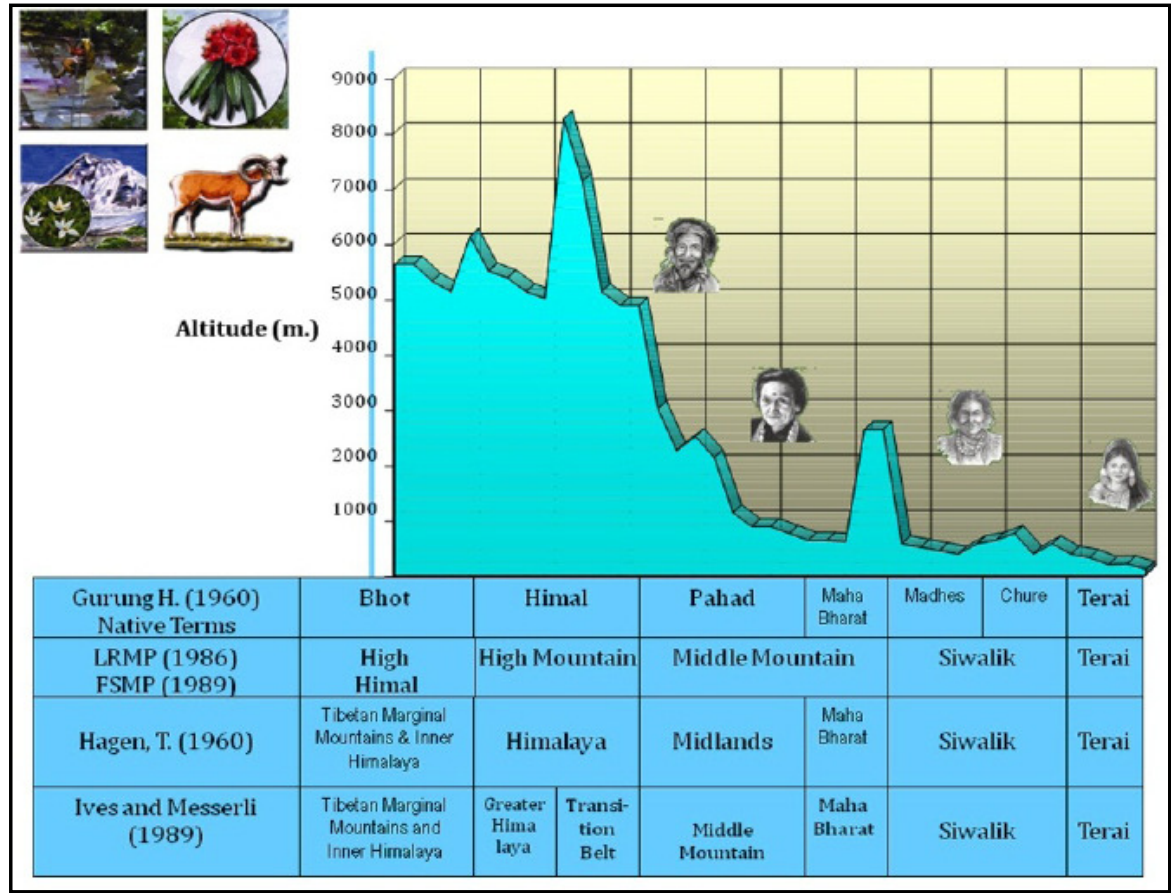

Source: Adapted from Shrestha 2011: 3

In Purcell's word, AIK/S can be 'the body of historically constituted (-emic) knowledge instrumental in the long-term adaptation of human groups to the biophysical environment' (260). It is the knowledge which is rooted and embedded in (indigenous to) the rurally located and socio-economically colonized, marginalized or underprivileged groups within Third/Fourth World Societies. A vast chunk of indigenous populations comprising more than $90 \%$ in Nepal, are no such exception of marginalization.

According to World Intellectual Property Organization (WIPO), AIK/S is often referred to as Traditional Knowledge (TK also known as ITK = indigenous technical knowledge system cf. Saul 136) and 'encompasses the content or substance of traditional know-how, innovations, information, practices, skills and learning of TK Systems such as traditional agricultural, environmental or medicinal knowledge'. 


\section{Crossing the Border: International Journal of Interdisciplinary Studies}

Such knowledge, developed from experience gained over time and adapted to the local culture and environment, has always played-and still plays-an important role in the daily lives of the majority of people globally and is considered to be an essential part of cultural identities. This reality should be reflected, considered and included in our national education curriculum.

It is very important to the food or economic security and health of millions of people in the developing (e.g., Nepal), and even developed countries. Moreover, such knowledge of the IPs or ethnic peoples (see Figure 1 and Table 1), which must be honored by the world (including Nepal), like knowledge developed after long research. Therefore, this knowledge must have rights like new knowledge gained by Research Company (RC) that patents its outcomes. For this, such knowledge or skills must be identified, recognized and documented through both formal and informal processes like Biodiversity Registration (BR) and in Nepal's context by including in national curriculum in technical and higher education.

Similarly, every country has to make the law concerning BR for such knowledge preservation and they should have to follow and respect international laws and declarations like the Convention on Biological Diversity (CBD 8 (j) Bhattarai 18-20) and their objectives for Indigenous Peoples (IPs) or ethnic groups' welfare. Also education and trainings on such skills should be provided to economically enhance and preserve for sustainable developmental efforts. These knowledge/skills are not only significant from economic point of view but have socio-cultural as well as political significance in their construction of identity. Due to this crucial cause, this paper has made an inventory of indigenous knowledge/skills (Table 1) of the officially recognized and ye-t-to-be-recognized IPs in Nepal.

Table 1: Inventory of indigenous skills/trades

\begin{tabular}{|c|c|c|c|}
\hline SN & IPs' Groups & Indigenous Skills/Trade Inventory & Economic Purpose \\
\hline \multicolumn{4}{|c|}{ 1. Eastern Mountain } \\
\hline 1. & Kulung & $\begin{array}{l}\Delta \text { Rokchokhi 'allo-related products' } \\
\Delta \text { Bamboo products } \\
\Delta \text { Gift items (made by orchid 'Sunakhari') }\end{array}$ & $\Delta$ Rokchokhi 'allo-related products' \\
\hline 2. & Lhomi & $\begin{array}{l}\Delta \text { Den-takken 'local carpet production' } \\
\Delta \text { Mentok 'local herbal collection' } \\
\Delta \text { Local bamboo products } \\
\Delta \text { Wooden crafts and utensils }\end{array}$ & $\begin{array}{l}\Delta \text { Den-takken 'local carpet production' } \\
\Delta \text { Mentok 'local herbal collection' } \\
\Delta \text { Local bamboo products }\end{array}$ \\
\hline 3. & Lohorung & $\begin{array}{l}\Delta \text { Saruwa 'local beer-making skill' } \\
\Delta \text { Allo Che 'cloth-making' } \\
\Delta \text { Khabek 'beer-making chemical' } \\
\Delta \text { Varieties of carpet by local materials like grasses } \\
\Delta \text { Cultural attire making (thread spinning) } \\
\Delta \text { Bamboo products }\end{array}$ & $\begin{array}{l}\Delta \text { Allo Che 'cloth-making' } \\
\Delta \text { Varieties of carpet by local materials like } \\
\text { grasses } \\
\Delta \text { Bamboo products }\end{array}$ \\
\hline 4. & Sherpa & $\begin{array}{l}\Delta \text { Singhi Nawa 'forest preserving skill' } \\
\Delta \text { Carpet products of local wool } \\
\Delta \text { Herbal medicine } \\
\Delta \text { Bamboo products }\end{array}$ & $\begin{array}{l}\Delta \text { Carpet products of local wool } \\
\Delta \text { Herbal medicine } \\
\Delta \text { Bamboo products }\end{array}$ \\
\hline 5. & Topkegola & $\begin{array}{l}\Delta \text { Radhi and Thumsi 'local carpet of local wool' } \\
\Delta \text { herbal, chiraito and its seed } \\
\Delta \text { Chhurpi milk products } \\
\Delta \text { potato chips } \\
\Delta \text { Bamboo products }\end{array}$ & $\begin{array}{l}\Delta \text { Radhi and Thumsi 'local carpet of local wool' } \\
\Delta \text { herbal, chiraito and its seed } \\
\Delta \text { milk products (chhurpi) } \\
\Delta \text { potato chips }\end{array}$ \\
\hline 6. & Walung & $\begin{array}{l}\Delta \text { Amchi 'local herbalist' } \\
\Delta \text { Kamlo/Liu 'local carpet of local wool' } \\
\Delta \text { Chyogchyi 'local carpentry' } \\
\Delta \text { Shompa 'local stockings made by males' } \\
\Delta \text { Thangka painting }\end{array}$ & $\begin{array}{l}\Delta \text { Amchi 'local herbalist' } \\
\Delta \text { Kamlo/Liu 'local carpet of local wool' } \\
\Delta \text { Chyogchyi 'local carpentry' }\end{array}$ \\
\hline
\end{tabular}


INCLUSIVE CURRICULUM

\begin{tabular}{|c|c|c|c|}
\hline 7. & Yamphu & $\begin{array}{l}\Delta \text { Bamboo products } \\
\Delta \text { Traditional cloth making }\end{array}$ & $\Delta$ Bamboo products \\
\hline & Thudam & $\begin{array}{l}\Delta \text { Amchi 'local herbalist' } \\
\Delta \text { Kamlo/Liu 'local carpet of local wool' } \\
\Delta \text { Chyogchyi 'local carpentry' } \\
\Delta \text { Shompa 'local stockings made by males' } \\
\Delta \text { Thangka painting }\end{array}$ & $\begin{array}{l}\Delta \text { Amchi 'local herbalist' } \\
\Delta \text { Kamlo/Liu 'local carpet of local wool' } \\
\Delta \text { Chyogchyi 'local carpentry' }\end{array}$ \\
\hline \multicolumn{4}{|c|}{ 2. Central Mountain } \\
\hline 8. & Aathpariya & $\begin{array}{l}\Delta \text { Mekhli-Chhitasim 'local cloth-making' } \\
\Delta \text { Bamboo products }\end{array}$ & $\begin{array}{l}\Delta \text { Mekhli-Chhitasim 'local cloth-making' } \\
\Delta \text { Bamboo products }\end{array}$ \\
\hline 9. & Hyolmo & $\begin{array}{l}\Delta \text { Singso 'carpentry' } \\
\Delta \text { Bamboo products } \\
\Delta \text { Thangka paintings } \\
\Delta \text { Chhyuba-Tehn-Bhrumsi 'local products of wool' }\end{array}$ & $\begin{array}{l}\Delta \text { Singso 'carpentry' } \\
\Delta \text { Bamboo products } \\
\Delta \text { Chhyuba-Tehn-Bhrumsi 'local products of } \\
\text { wool' } \\
\Delta \text { Lokta 'local fibre' production }\end{array}$ \\
\hline 10. & Jirel & $\begin{array}{l}\Delta \text { Lokta 'local fibre' production } \\
\Delta \text { Bamboo products }\end{array}$ & $\Delta$ Lokta 'local fibre' production \\
\hline 11. & Tamang & $\begin{array}{l}\Delta \text { Kwan Raba 'cloth making' } \\
\Delta \text { Chhaige 'baskets and ropes' } \\
\Delta \text { Syosyo Shengba 'hand-made paper' } \\
\Delta \text { Sing Thaba 'wooden craft' } \\
\Delta \text { Thangka 'cultural paint' } \\
\Delta \text { Marcha 'Herbal yeast' } \\
\Delta \text { Bamboo products }\end{array}$ & $\begin{array}{l}\Delta \text { Kwan Raba 'cloth making' } \\
\Delta \text { Sing Thaba 'wooden craft' } \\
\Delta \text { Thangka 'cultural paint' } \\
\Delta \text { Bamboo products }\end{array}$ \\
\hline 12. & Tha(ng)mi & $\begin{array}{l}\Delta \text { Ding khani 'stone mine' } \\
\Delta \text { Bhangra 'cloth of Allo' } \\
\Delta \text { Bamboo product }\end{array}$ & $\begin{array}{l}\Delta \text { Bhangra 'cloth of Allo' } \\
\Delta \text { Bamboo product }\end{array}$ \\
\hline \multicolumn{4}{|c|}{ 3. Western Mountain } \\
\hline 13. & Lhopa & $\begin{array}{l}\Delta \text { Kedin 'cultural attire for female' } \\
\Delta \text { Ten 'local carpet' } \\
\Delta \text { Amchi 'local herbalist' }\end{array}$ & $\begin{array}{l}\Delta \text { Kedin 'cultural attire for female' } \\
\Delta \text { Ten 'local carpet' } \\
\Delta \text { Amchi 'local herbalist' }\end{array}$ \\
\hline 14. & Thakali & $\begin{array}{l}\Delta \text { Dhumchan 'carpet made up of local wool' } \\
\Delta \text { Pulu 'cap made up of pasmina wool' } \\
\Delta \text { Cho 'thread', Bhaleshyu 'socks made up of wool' } \\
\Delta \text { Yashyu 'gloves made up of wool' } \\
\Delta \text { Phutum 'cultural attire of female made up of wool' } \\
\Delta \text { Amchi 'local herbalist' } \\
\Delta \text { Ngakpa 'local neurologist' }\end{array}$ & $\begin{array}{l}\Delta \text { Woolen products } \\
\Delta \text { Amchi 'local herbalist' }\end{array}$ \\
\hline \multicolumn{4}{|c|}{ 4. Mid -Western Mountain } \\
\hline 15. & Dolpo & $\begin{array}{l}\Delta \text { Amchi 'local herbalist' } \\
\Delta \text { Kamlo/Liu 'local carpet of local wool' } \\
\Delta \text { Chyogchyi 'local carpentry' } \\
\Delta \text { Shompa 'local stockings made by males' } \\
\Delta \text { Thangka painting } \\
\Delta \text { Bamboo products }\end{array}$ & $\begin{array}{l}\Delta \text { Amchi 'local herbalist' } \\
\Delta \text { Kamlo/Liu 'local carpet of local wool' }\end{array}$ \\
\hline \multicolumn{4}{|c|}{ 5. Far-Western Mountain } \\
\hline 16. & Bhote & $\begin{array}{l}\Delta \text { Radhi and Thumsi 'local carpet' } \\
\Delta \text { Amchi 'local herbalist' } \\
\Delta \text { Bamboo products }\end{array}$ & $\begin{array}{l}\Delta \text { Radhi and Thumsi 'local carpet' } \\
\Delta \text { Amchi 'local herbalist' }\end{array}$ \\
\hline 17. & Byasi/Sauka & $\begin{array}{l}\Delta \text { Tsav pae 'local carpet' } \\
\Delta \text { Chedar 'local cloth making' } \\
\Delta \text { Dangre/Lama 'local herbalist like Amchi' }\end{array}$ & $\begin{array}{l}\Delta \text { Tsav pae 'local carpet' } \\
\Delta \text { Chedar 'local cloth making' } \\
\Delta \text { Dangre/Lama 'local herbalist like Amchi' }\end{array}$ \\
\hline 18. & Mugal & $\begin{array}{l}\Delta \text { Ralpa 'local carpet of wool' production' } \\
\Delta \text { Dhau 'local Bhakhkhu' } \\
\Delta \text { Nhanak 'women shawl' } \\
\Delta \text { Amchi 'local herbalist' }\end{array}$ & $\begin{array}{l}\Delta \text { Woolen products } \\
\Delta \text { Amchi 'local herbalist' }\end{array}$ \\
\hline \multicolumn{4}{|c|}{ 6. Eastern Hill } \\
\hline 19. & Lepcha & $\begin{array}{l}\Delta \text { Karbomu 'metal work' production } \\
\Delta \text { Damrapu 'cloth making' } \\
\Delta \text { Lahuk 'wood work' production } \\
\Delta \text { Karmomu 'female jewellery' production }\end{array}$ & $\begin{array}{l}\Delta \text { Damrapu 'cloth making' } \\
\Delta \text { Lahuk 'wood work' production }\end{array}$ \\
\hline
\end{tabular}




\section{Crossing the Border: International Journal of Interdisciplinary Studies}

\begin{tabular}{|c|c|c|c|}
\hline 20. & $\begin{array}{l}\text { Kiranti- } \\
\text { Yakthung } \\
\text { (Limbu) }\end{array}$ & $\begin{array}{l}\Delta \text { Yangben 'lichen' preparation' } \\
\Delta \text { Kinema 'bacteria-fermented soybean' production } \\
\Delta \text { Tongba 'local beverage' production } \\
\Delta \text { Khe?sung 'yeast' production, } \\
\Delta \text { Khisukwa 'traditional bag' production } \\
\Delta \text { Bamboo products }\end{array}$ & $\begin{array}{l}\Delta \text { Kinema 'bacteria-fermented soybean' pro- } \\
\text { duction } \\
\Delta \text { Khisukwa 'traditional bag' production } \\
\Delta \text { Bamboo products }\end{array}$ \\
\hline 21. & $\begin{array}{l}\text { Kiranti-Thulung } \\
\text { Kiranti-Rodung } \\
\text { Kiranti-Kirawa } \\
\text { Kiranti-Mewahang } \\
\text { Kiranti-Sampang } \\
\text { Kiranti-Khaling }\end{array}$ & $\begin{array}{l}\Delta \text { Kinema 'bacteria-fermented soybean' production } \\
\Delta \text { Tongba 'beverage' production } \\
\Delta \text { Marcha 'yeast' production } \\
\Delta \text { Champhuri 'cultural attire' production } \\
\Delta \text { Allo, the Himalayan Giant Nettle (Girardinia } \\
\text { Diversifoli) production } \\
\Delta \text { Bamboo crafts and products }\end{array}$ & $\begin{array}{l}\Delta \text { Kinema 'bacteria-fermented soybean' pro- } \\
\text { duction } \\
\Delta \text { Champhuri 'cultural attire' production } \\
\Delta \text { Allo, the Himalayan Giant Nettle (Girardinia } \\
\text { Diversifoli) production } \\
\Delta \text { Bamboo crafts and products }\end{array}$ \\
\hline 22. & $\begin{array}{l}\text { Kiranti- } \\
\text { Yakkha }\end{array}$ & $\begin{array}{l}\Delta \text { Yangben 'lichen' preparation } \\
\Delta \text { Kinema 'bacteria-fermented soybean' production } \\
\Delta \text { Tongba 'local beverage' production } \\
\Delta \text { Thawa 'yeast' production } \\
\Delta \text { Bamboo products }\end{array}$ & $\begin{array}{l}\Delta \text { Kinema 'bacteria-fermented soybean' pro- } \\
\text { duction } \\
\Delta \text { Bamboo products }\end{array}$ \\
\hline \multicolumn{4}{|c|}{ 7. Central Hill } \\
\hline 23. & $\begin{array}{l}\text { Chepang/ } \\
\text { Praja }\end{array}$ & $\begin{array}{l}\Delta \text { Pande 'local herbalist' } \\
\Delta \text { Jabi 'fibre bag production' } \\
\Delta \text { bamboo and asparagus products } \\
\Delta \text { Butter production of Bassiya Butyracea } \\
\Delta \text { Bee-keeping and honey hunting }\end{array}$ & $\begin{array}{l}\Delta \text { bamboo and asparagus products } \\
\Delta \text { bee-keeping and honey hunting }\end{array}$ \\
\hline 24. & Danuwar & $\begin{array}{l}\Delta \text { Mujela 'basket' production of reed } \\
\Delta \text { Fishing net }\end{array}$ & $\Delta$ Mujela 'basket' production of reed \\
\hline 25. & Hayu & $\Delta$ local drum products of local wood & $\Delta$ local drum products of local wood \\
\hline 26. & Majhi & $\Delta$ Boating, Fishing and Fishery & $\Delta$ Boating, Fishing and Fishery \\
\hline 27. & $\begin{array}{l}\text { Newar } \\
\text { Kayastha }\end{array}$ & $\begin{array}{l}\Delta \text { Chayagubhada 'pottery' } \\
\Delta \text { Haku Tapuli 'cultural cap' (Bhadgaule Topi) } \\
\Delta \text { Thajya Thaegu 'local spinning' }\end{array}$ & $\begin{array}{l}\Delta \text { Chayagubhada 'pottery' } \\
\Delta \text { Haku Tapuli 'cultural cap' (BhadgauleTopi) } \\
\Delta \text { Thajya Thaegu 'local spinning' }\end{array}$ \\
\hline 28. & Pahari & $\begin{array}{l}\Delta \text { Statue/icon making } \\
\Delta \text { Cane split/root/stem } \\
\Delta \text { Bamboo products }\end{array}$ & $\begin{array}{l}\Delta \text { Statue/icon making } \\
\Delta \text { Bamboo products }\end{array}$ \\
\hline 29. & $\begin{array}{l}\text { Kiranti-Kõits } \\
(\text { Sunuwar/ } \\
\left.\text { Mukhiya }^{3}\right)\end{array}$ & $\begin{array}{l}\Delta \text { Phenerelphu 'neck-kerchief' knitting } \\
\Delta \text { Klatori 'waist-belt' knitting } \\
\Delta \text { Ple:kas 'memory knot' production } \\
\Delta \text { Deli-Phipi 'bamboo products' } \\
\Delta \text { Binayo 'bamboo musical instrument' product }\end{array}$ & $\begin{array}{l}\Delta \text { Phenerelphu 'neck-kerchief' knitting } \\
\Delta \text { Deli-Phipi 'bamboo products' } \\
\Delta \text { Binayo 'bamboo musical instrument' product }\end{array}$ \\
\hline 30. & Surel & $\Delta$ Bamboo products & $\Delta$ Bamboo products \\
\hline \multicolumn{4}{|c|}{ 8. Western Hill } \\
\hline 31. & Brahmu/ Baramo & $\Delta$ Masonry & $\Delta$ Masonry \\
\hline 32. & $\begin{array}{l}\text { Chhantyal/ } \\
\text { Chhantel }\end{array}$ & $\Delta$ Allo and bamboo products & $\Delta$ Allo and bamboo products \\
\hline 33. & Darai & $\begin{array}{l}\Delta \text { Marcha 'yeast making' } \\
\Delta \text { Bamboo products }\end{array}$ & $\Delta$ Bamboo products \\
\hline 34. & Dura & $\begin{array}{l}\Delta \text { Radi 'woolen carpet' } \\
\Delta \text { Lukuni 'woolen jacket' } \\
\Delta \text { Kũ 'bamboo umbrella' } \\
\Delta \text { Honey-hunting } \\
\Delta \text { Bee-keeping } \\
\Delta \text { Bamboo products } \\
\Delta \text { Cultural gift item (Jantar) }\end{array}$ & $\begin{array}{l}\Delta \text { Bee-keeping } \\
\Delta \text { Bamboo products } \\
\Delta \text { Cultural gift item (Jantar) }\end{array}$ \\
\hline 35. & Gharti/ Bhujel & $\begin{array}{l}\Delta \text { Bamboo products } \\
\Delta \text { Herbal medicine }\end{array}$ & $\Delta$ Bamboo products \\
\hline 36. & Gurung & $\begin{array}{l}\Delta \text { Radi 'woolen carpet' } \\
\Delta \text { Lukuni 'woolen jacket' } \\
\Delta \text { Kú 'bamboo umbrella' } \\
\Delta \text { Takroye Chya 'wooden bird' } \\
\Delta \text { Honey-hunting } \\
\Delta \text { Bee-keeping } \\
\Delta \text { Bamboo products }\end{array}$ & $\begin{array}{l}\Delta \text { Radi 'woolen carpet' } \\
\Delta \text { Lukuni 'woolen jacket' } \\
\Delta \text { Bamboo products }\end{array}$ \\
\hline
\end{tabular}


INCLUSIVE CURRICULUM

\begin{tabular}{|c|c|c|c|}
\hline 37. & Kusunda & $\Delta$ Herbal collection & $\Delta$ Herbal collection \\
\hline 38. & Magar & $\begin{array}{l}\Delta \text { Khurpeto, Gyado, Ghalek, Madal, Khaijadi Production } \\
\Delta \text { Bamboo products esp. winnowing objects }\end{array}$ & $\Delta$ Bamboo products \\
\hline 39. & Kumal & $\Delta$ Pottery & $\Delta$ Pottery \\
\hline 40. & $\begin{array}{l}\text { Larke }(\mathrm{Nup}(\mathrm{b}) \\
\text { ri) }\end{array}$ & $\begin{array}{l}\Delta \text { Amchi 'local herbalist' } \\
\Delta \text { Pangkep 'cloth production' } \\
\Delta \text { Janduk 'local carpet' production } \\
\Delta \text { Sinjuwa 'wooden craft' } \\
\Delta \text { Tojuwa 'stone craft' }\end{array}$ & $\begin{array}{l}\Delta \text { Amchi 'local herbalist', } \\
\Delta \text { Sinjuwa 'wooden craft', }\end{array}$ \\
\hline 41. & Baragaũle & $\begin{array}{l}\Delta \text { Keden 'female attire made up of local wool' } \\
\Delta \text { Pangden 'attire for female in marriage made up } \\
\text { of local wool' }\end{array}$ & $\begin{array}{l}\Delta \text { Keden 'female attire made up of local wool', } \\
\Delta \text { Pangden 'attire for female in marriage made } \\
\text { up of local wool' }\end{array}$ \\
\hline \multicolumn{4}{|c|}{ 9. Mid Western Hill } \\
\hline 42. & Raute & $\Delta$ Koshi, Madhus, Jhuma 'wooden utensils' & $\Delta$ Koshi, Madhus, Jhuma 'wooden utensils' \\
\hline \multicolumn{4}{|c|}{ 10. Far Western Hill } \\
\hline & \multicolumn{3}{|c|}{ This region is also inhabited by the Rautes } \\
\hline \multicolumn{4}{|c|}{ 11. Eastern Terai } \\
\hline 43. & Dhanuk & $\begin{array}{l}\Delta \text { Dali 'straw-basket making' } \\
\Delta \text { Bhuja 'local food' }\end{array}$ & $\Delta$ Dali 'straw-basket making' \\
\hline 44. & Dhimal & $\begin{array}{l}\Delta \text { Mhoti 'herbal yeast production', } \\
\Delta \text { Tana 'looming' } \\
\Delta \text { Dungri 'fish-net' } \\
\Delta \text { Bona 'women's cultural attire' } \\
\Delta \text { Tepana 'cultural belt for women' }\end{array}$ & $\begin{array}{l}\Delta \text { Tana 'looming' } \\
\Delta \text { Dungri 'fish-net' } \\
\Delta \text { Bona 'women's cultural attire' } \\
\Delta \text { Tepana 'cultural belt for women' }\end{array}$ \\
\hline 45. & Gangai & $\begin{array}{l}\Delta \text { Dali 'straw-basket making' } \\
\Delta \text { Bhuja 'local food' }\end{array}$ & $\Delta$ Dali 'straw-basket making' \\
\hline 46. & $\begin{array}{l}\text { Jhãgad/ } \\
\text { Dhagar }\end{array}$ & $\begin{array}{l}\Delta \text { Pitri 'mattress made up of local fibre' } \\
\Delta \text { Dauda 'basket made up of local reed' } \\
\Delta \text { Chalki 'made up of local plant' } \\
\Delta \text { Chauki-Jalli-Pahi-Jalli 'fish net made up of thread' } \\
\Delta \text { Ukhudi 'wooden mortar' } \\
\Delta \text { Baksa 'wooden box' } \\
\Delta \text { Pitonji-Ghumri 'garland for curing allergy' }\end{array}$ & $\begin{array}{l}\Delta \text { Pitri 'mattress made up of local fibre' } \\
\Delta \text { Dauda 'basket made up of local reed' } \\
\Delta \text { Chalki 'made up of local plant' } \\
\Delta \text { Chauki-Jalli-Pahi-Jalli 'fish net made up of } \\
\text { thread', } \\
\Delta \text { Pitonji-Ghumri 'garland for curing allergy' }\end{array}$ \\
\hline 47. & Kewat & $\Delta$ Baskets and carpets by local straw and grasses & $\Delta$ Baskets and carpets by local straw and grasses \\
\hline 48. & Kisan & $\begin{array}{l}\Delta \text { Clay pottery } \\
\Delta \text { Taripatta 'handicraft' products }\end{array}$ & $\Delta$ Taripatta 'handicraft' products \\
\hline 49. & $\begin{array}{l}\text { Koch (Ra- } \\
\text { jbamsi) }\end{array}$ & $\begin{array}{l}\Delta \text { Bhakra 'local jute carpet' } \\
\Delta \text { Petani 'cloth making' } \\
\Delta \text { Jauli 'local back' production }\end{array}$ & $\begin{array}{l}\Delta \text { Bhakra 'local jute carpet' } \\
\Delta \text { Petani 'cloth making' } \\
\Delta \text { Jauli 'local back' production }\end{array}$ \\
\hline 50. & Kumal & $\Delta$ Pottery & $\Delta$ Pottery \\
\hline 51. & Kumhar & $\Delta$ Baskets and carpets by local straw and grasses & $\Delta$ Baskets and carpets by local straw and grasses \\
\hline 52. & Mallaha & $\Delta$ Fishing, boating, fishing net making & $\Delta$ Fishing, boating, fishing net making \\
\hline 53. & Meche & $\Delta$ Dokhna-Aronai 'local handloom' & $\Delta$ Dokhna-Aronai 'local handloom' \\
\hline 54. & Munda $^{4}$ & $\begin{array}{l}\Delta \text { Local handloom } \\
\Delta \text { Baskets and carpets by straw and grasses }\end{array}$ & $\begin{array}{l}\Delta \text { Local handloom } \\
\Delta \text { Baskets and carpets by straw and grasses }\end{array}$ \\
\hline 55. & Rajbamsi & $\begin{array}{l}\Delta \text { Bhakra 'local jute carpet' } \\
\Delta \text { Petani 'cloth making' } \\
\Delta \text { Jauli 'local bag' production }\end{array}$ & $\begin{array}{l}\Delta \text { Bhakra 'local jute carpet' } \\
\Delta \text { Petani 'cloth making' } \\
\Delta \text { Jauli 'local bag' production }\end{array}$ \\
\hline 56. & Satar/ Santhal & $\begin{array}{l}\Delta \text { Clay pottery } \\
\Delta \text { Taripatta 'handicraft' products }\end{array}$ & $\Delta$ Taripatta 'handicraft' products \\
\hline 57. & Tajpuriya & $\begin{array}{l}\Delta \text { Dhakra 'local jute carpet making' } \\
\Delta \text { Bjuja-Bhakka 'indigenous local dishes' }\end{array}$ & $\begin{array}{l}\Delta \text { Dhakra 'local jute carpet making' } \\
\Delta \text { Bjuja-Bhakka 'indigenous local dishes' }\end{array}$ \\
\hline \multicolumn{4}{|c|}{ Tharu (Khawas) } \\
\hline \multicolumn{4}{|c|}{ 12. Central Terai } \\
\hline 58. & Amat & $\begin{array}{l}\Delta \text { Local handloom } \\
\Delta \text { Basket and carpet by straw and grasses }\end{array}$ & $\begin{array}{l}\Delta \text { Local handloom } \\
\Delta \text { Basket and carpet by straw and grasses }\end{array}$ \\
\hline
\end{tabular}


Crossing the Border: International Journal of Interdisciplinary Studies

\begin{tabular}{|c|c|c|c|}
\hline 59. & Kurmi & $\begin{array}{l}\Delta \text { Local handloom } \\
\Delta \text { Basket and carpet by straw and grasses }\end{array}$ & $\begin{array}{l}\Delta \text { Local handloom } \\
\Delta \text { Basket and carpet by straw and grasses }\end{array}$ \\
\hline & \multicolumn{3}{|c|}{ Tharu indigenous people also live in this region } \\
\hline \multicolumn{4}{|c|}{ 13. Western Terai } \\
\hline 60. & Bote & $\begin{array}{l}\Delta \text { Fishing, Fishery } \\
\Delta \text { Boating }\end{array}$ & $\begin{array}{l}\Delta \text { Fishing, Fishery } \\
\Delta \text { Boating }\end{array}$ \\
\hline & \multicolumn{3}{|c|}{ Tharu indigenous people also live in this region } \\
\hline \multicolumn{4}{|c|}{ 14. Mid Western Terai } \\
\hline 61. & $\begin{array}{l}\text { Pattharkatta/ } \\
\text { Kushbadiya }\end{array}$ & $\begin{array}{l}\Delta \text { Stone mortar } \\
\Delta \text { Medicine products of jackal } \\
\Delta \text { Threads and nets products of jutes }\end{array}$ & $\begin{array}{l}\Delta \text { Stone mortar } \\
\Delta \text { Threads and nets products of jutes }\end{array}$ \\
\hline 62. & Tharu & $\begin{array}{l}\Delta \text { Jali 'fishnet making' } \\
\Delta \text { Sopi 'rain-coat like object making' } \\
\Delta \text { Deli 'bamboo object for storing food-grains' }\end{array}$ & $\begin{array}{l}\Delta \text { Jali 'fishnet making' } \\
\Delta \text { Sopi 'rain-coat like object making' } \\
\Delta \text { Deli 'bamboo object for storing food-grains' }\end{array}$ \\
\hline \multicolumn{4}{|c|}{ 15. Far Western Terai } \\
\hline 63. & Raji & $\begin{array}{l}\Delta \text { Boating, fishing, } \\
\Delta \text { Herbal and leaf collection }\end{array}$ & $\begin{array}{l}\Delta \text { Boating, fishing, } \\
\Delta \text { Herbal and leaf collection }\end{array}$ \\
\hline & \multicolumn{3}{|c|}{ Far Western Terai is the original homeland of the Tharu indigenous people } \\
\hline
\end{tabular}

Source: Adapted from Rapacha and Thulung Rai 2013: 19-23

Diverse types and forms of AIK/S in Table 1 above was identified and documented through individual consultations, related reports and literature(s) of the indigenous peoples residing in different parts of the country as shown in Figure 1 earlier. Furthermore, the table shows that many of the indigenous peoples either living in geographical proximity or far from one another have similar types of indigenous skills. For example, many of the indigenous groups, especially in the hills, have skills and knowledge of making varieties of bamboo products/goods. Similarly, woolen carpets, local handloom, local herbal medicine, cultural attire production, carpets made up of local materials including grasses and straw, wooden crafts and many more types of skills are common among many of the indigenous peoples.

To be more specific, herbal medicine, woolen blankets, carpets and cloths, varieties of bamboo products wooden crafts are common among the indigenous peoples in the mountain regions. While, varieties of bamboo products, handloom, cultural attire production, local beer making, wooden utensils are common for most of the indigenous peoples in the hills. Also, local food varieties, carpets and blankets by straw and other local raw materials, handloom, thread spinning are some common indigenous skills for the peoples in the Terai region.

The similarity and dissimilarity in the indigenous skills and knowledge indicate that our national curriculum should redress geographic locations, linguistic and cultural backgrounds of the students such as the knowledge of Amchi is much more relevant to the students of the Mountain regions. The same case applies with the availability of natural resources, e.g., Cordyceps sinensis (caterpillar fungus or Yarsa as in Photo 1 below) and other related biodiversity in diverse ecological belts (Figure 1) and development regions of the country. It should highly be reflected, recognized and included in the national curriculum of technical and higher education so that our education system and invention can be unique in the world to our own context and situation. 


\section{INCLUSIVE CURRICULUM}

\section{INVENTORY OF YARSA, AMCHI, ETHNOMEDICINE AND ECONOMIC PO- TENTIALITY}

National information and knowledge on our multitude of treasures seem to be very meagre-as aboriginal history unrecorded and forgotten. One of such treasures in our of late information is yarsa-an indigenous Nepal Himalaya ethnomedicinal (having its roots in ethnobotany) herb used as tonic, aphrodisiac and also used to cure other diseases. It has hit our national newspaper-pages due to its millions of worth. Choppers have been hired to ferry yarsa ${ }^{5}$ to the capital city for supply in raw forms to foreign nations like India, China, Singapore, US, Germany, France, Italy Czech Republic and so forth. Yarsa's local economic value in its raw form is up to NRs. 2 million per $\mathrm{kg}$ and its international market price is still unknown. We are also unknown to its further processing in those foreign medical laboratories and its actual value whether economic or medicinal. Our local Amchi knowledge (see also Koirala 5) on such valuable herb in our university medical departments, is yet awaiting to be recognized and included for further knowledge and sustainable economic-gain. Nepal had exported raw herbal products to the outside world worth about NRs. 805.37 million in 2011 according to Trade and Export Promotion Centre (TEPC qtd in Rapacha and Thulung Rai 38-39). This figure might have increased in recent years as in the case of handicrafts exports (see Table 2).

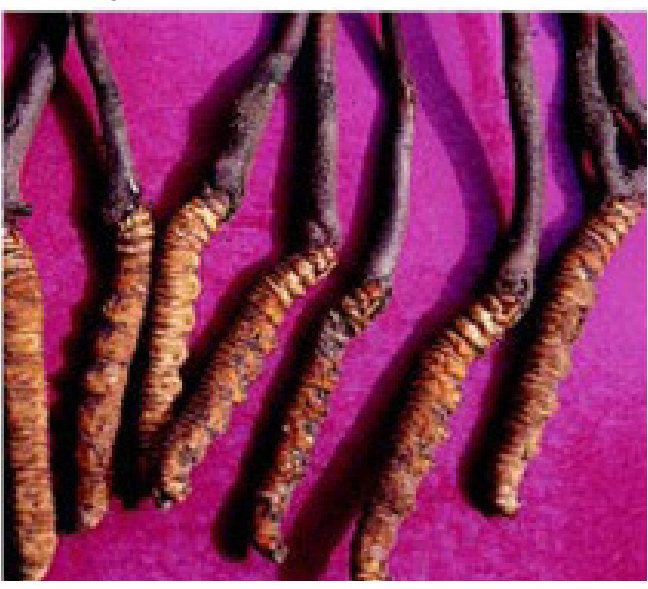

Photo Plate 1: Yarsa Lama et al 2001: 56

Besides yarsa, many other ethnomedicinal herbs like potato yam, wild yam, lichen, phyllanthus emblica, soap nut, satuwa (love apple) ${ }^{6}$ etc. (also see Lama, Ghimire Aumeeruddy-Thomas 2001 for more such herbs in Dolpo) and have almost equal medicinal and economic potentiality since the indigenous local Gyushi believe(s) that "Every plant has a medicinal value once its taste and potency are known". Additionally, plants have linguistic and cultural significance amongst all indigenous peoples, e.g., in Kiranti-Kõits people's culture, the mythological story of Khekhephu Bospat 'holiest flower' or oroxylum indicum (Linnaeus) Kurz 'Nepalese/Indian trumpet flower' (Rapacha 13-32) has cultural significance of their identity.

Moreover, the Põibo-Gyami (Kiranti-Kõits words for 'male and female shamans') in the hills have equally competent innovative knowledge about plants and traditional healing system or ethnopsychoatric (see Peters 20) knowledge unrecorded. Of course, it is our dire need to explore and innovate such unfathomable depth of knowledge by making our technical and higher education curriculum much more diverse and inclusive.

\section{INVENTORY OF HANDICRAFTS AND ECONOMIC POTENTIALITY}

In our inventories of indigenous knowledge skills/trades in Table 1 earlier, one can easily observe diverse skills in handicrafts productions supporting for gift economy and for exports to local and international markets as listed in Table 2 below. 


\section{Crossing the Border: International Journal of Interdisciplinary Studies}

Table 2: Inventory of handicrafts and national income

\begin{tabular}{|c|c|c|c|c|c|c|c|}
\hline SN & Non-Textile Products & $\begin{array}{l}\text { Exported } \\
\text { Amount } \\
\text { (in NRs) }\end{array}$ & $\%$ & SN & Textile Products & $\begin{array}{l}\text { Exported } \\
\text { Amount } \\
\text { (in NRs) }\end{array}$ & $\%$ \\
\hline 1 & Bamboo Products & 2042557 & 0.18 & 1 & Allo Goods & 814426 & 0.08 \\
\hline 2 & Crystal Products & 4582502 & 0.40 & 2 & Dhaka Products & 1610735 & 0.17 \\
\hline 3 & Stone Craft & 5235045 & 0.46 & 3 & Misc. Textile Products & 8868347 & 0.91 \\
\hline 4 & Thanka (Paubha) & 5862994 & 0.51 & 4 & Hemp Goods & 11346891 & 1.16 \\
\hline 5 & Beads Items & 6327248 & 0.56 & 5 & Silk Products & 57729245 & 5.93 \\
\hline 6 & Plastic Items & 8713223 & 0.77 & 6 & Cotton Goods & 71925124 & 7.38 \\
\hline 7 & Leather Goods & 8759971 & 0.77 & 7 & Felt Products & 229730809 & 23.58 \\
\hline 8 & Incense & 13528076 & 1.19 & 8 & Pashmina Products & 256149678 & 26.29 \\
\hline 9 & Ceramics Products & 20436205 & 1.79 & 9 & Woolen Goods & 336136114 & 34.50 \\
\hline 10 & Bone and Horn Products & 29938245 & 2.63 & & & & \\
\hline 11 & Miscellaneous Goods & 37520420 & 3.29 & & & & \\
\hline 12 & Glass Products & 39687124 & 3.49 & & & & \\
\hline 13 & Wood Craft & 46722183 & 4.10 & & & & \\
\hline 14 & Handmade Paper Products & 208467525 & 18.31 & & & & \\
\hline 15 & Silver Products & 247640851 & 21.75 & & & & \\
\hline \multirow[t]{2}{*}{16} & Metal Craft & 453328131 & 39.81 & & & & \\
\hline & Total & 1138792300 & 100.00 & & Total & 974311369.5 & 100.00 \\
\hline
\end{tabular}

Source: FHA (Federation of Handicraft Association) 2011 qtd in Rapacha and Thulung Rai 2013: 80

Broadly speaking, the two categories of products such as non-textile and textile listed in Table 2 has very potential economic gain in millions or even billion as the figure suggests. Most of these products except for leather goods and metal craft, are related with the indigenous knowledge and skills of the aboriginal people of Nepal residing in diverse regions (Figure 1) of Nepal. As compared to 2011 (Table 2), the economic growth of handicrafts exports to China and India in 2012-13 has amazingly increased up to NRs. 136.94 million and 61.13 million $^{7}$ respectively. Some of the selective components' knowledge and skills can be included in our curriculum for making it more participatory and inclusive.

\section{INVENTORY OF ARTIFACT AND ARCHAEOLOGY}

All indigenous peoples mentioned in Table 1 before have unique ways of producing objects or artifacts which have cultural and archaeological importance, e.g., the Kiranti-Kõits people's Plẽkās 'memory knot' (Photo Plate 1), Turung 'Bonbo/shaman drum' (Photo Plate 2) and Gũiduwa 'Shyãdar drum' (Photo Plate 3) and so on.

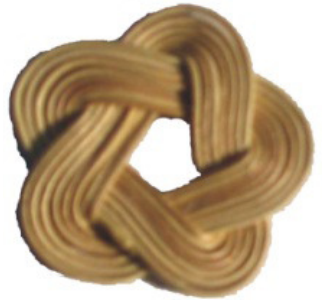

Photo Plate 2: Plẽkās 'memory knot'

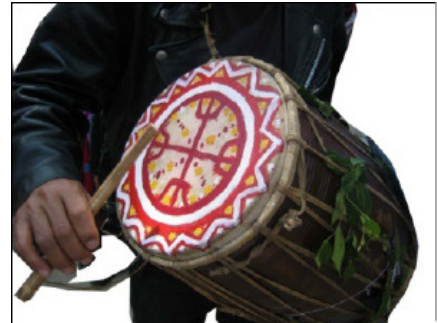

Photo Plate 3: Gũiduwa 'Shyãdar drum'-trident representing male and lotus-female

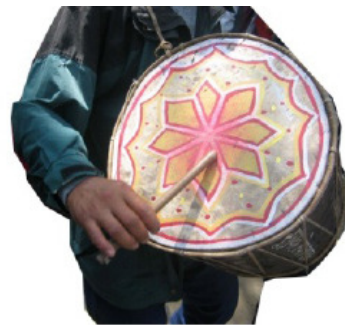

94 


\section{INCLUSIVE CURRICULUM}

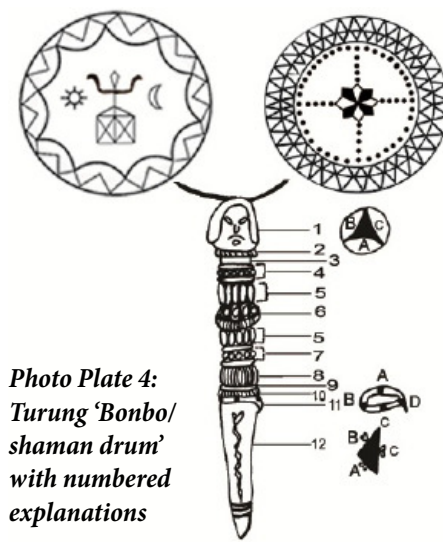

1. Piya 'head' (Kiranti-Kõits lexeme where Tsinge Lama, Nima and Urgin Tele are Tibetan lexemes)

Batsa 'support, neck' (Kiranti-Kõits lexeme)

3. Balā 'shoulder' (Kiranti-Kõits lexeme)

4. Tukse Mane 'straight shrine' (Kiranti-Kõits lexeme and Tibetan lexeme)

5. Lare Mane 'shrine' (Kiranti-Kõits and Tibetan lexeme)

6. Badzer Guru 'teacher/master' (Kiranti-Kõits and Tibetan lexeme being the last one from Sanskrit loan)

7. Mane Tsiter 'shrine' (Tibetan lexeme)

8. Tsatala* Darti 'six ladder land' (Indo-Aryan lexeme?)

9. Nag 'snake' (Buś in Kiranti-Kõits)

10. Pira 'mattress' (Tsingli in Kiranti-Kõits)

11. Batsher, Diyo, Sang, Murtsil (Diyo 'light' Indo-Aryan lexeme? and Tibetan/ Kiranti-Kõits lexeme?)

12. Murtsil (Tibetan or Kiranti-Kõits lexeme)

Note: Rapacha (2011: 18-19) has added more linguistic information after Fournier 1976

These artifacts in all Photo Plates 2-4 bear natural, supernatural and cosmological knowledge of the people (cf. Rapacha 18-19) having cultural and archaeological values. Such valuable artifacts with such knowledge have not yet been recognized and included in our national curriculum.

\section{INVENTORY OF THE BELIEF SYSTEMS, GENESIS AND WORLD VIEWS}

Till date our education curriculum has overfed generations of students mostly the western, dominant and power-centered cultural and religious world views by sidelining multi-belief systems, genesis and world views-an elite student or scholar hardly does intellectual-exercise in knowing them. Here we look at one of such inventories of Mugdum, from east Nepal (Rapacha 15), sometimes termed synonymously as Pelam 'method, system, procedure' (Chemjong and Nembang 2059: 278) originates from mundhukma 'to pray, recite incantation(s), utter the mystical verse quietly' [> (n.) Mundhum] in Kiranti-Yakthung Pan (ibid: 343) with some other linguistic variations as Mundum, Mundhum, Mukdum, Mudhdum and so forth depending on multilingual Kiranti linguistic communities of all three Kirant(i) areas-known as Wallo 'hither/near', Majh 'middle/mid/central' and Pallo 'Far' Kirant (cf. Pradhan 115). Scientifically, Mugdum is an integrated whole (see Figure 4) of plural components as drawn in cyclical Fig 2 above. It is mainly classified into two branches-known as Pesap (Blesisho in Kiranti-Kõits) 'written' and Thungsap (Warsisho in Kiranti-Kõits) 'spoken' in Kiranti-Yakthung Pan (Figure 2).
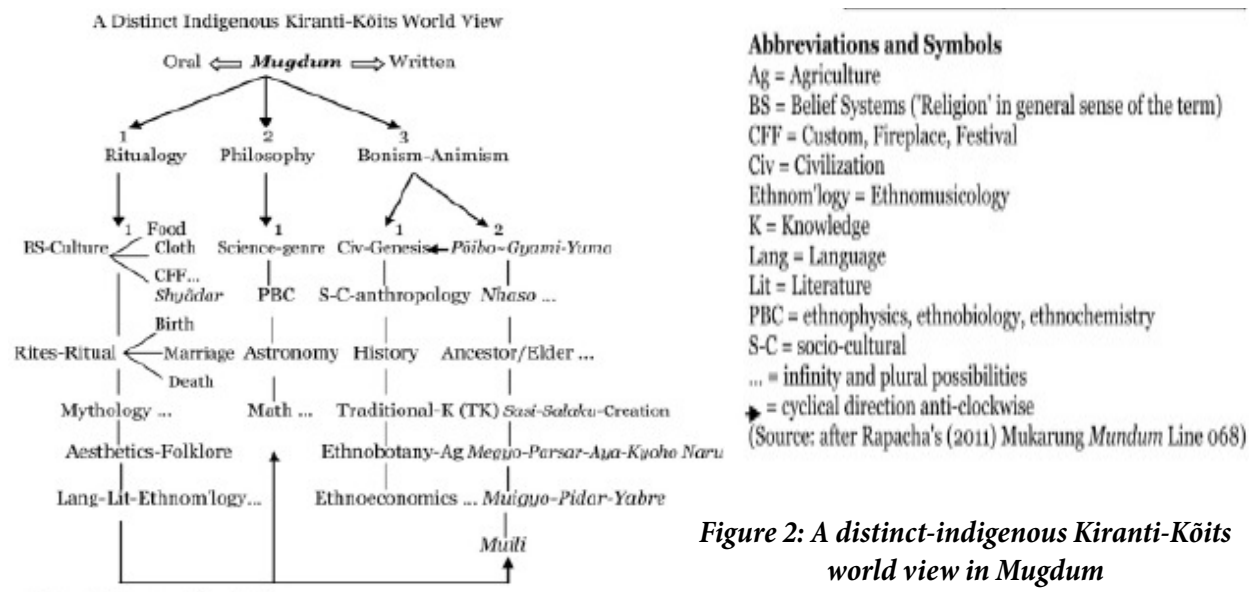

Figure 2: A distinct-indigenous Kiranti-Kõits world view in Mugdum 


\section{Crossing the Border: International Journal of Interdisciplinary Studies}

As shown in Figure 2, it is commonly a spiritual, rhythmic and shamanic form of scriptures. The Mugdum rituals and teachings are only used and performed by a KirantiKõits Nhaso 'religious teacher/s' or Põibo Gyami 'Bonbo/shamanic expert (also cf. Subba 102) both male and female. It is a blend of animism and ancestor worship meaning 'the power of knowledge, great strength and light' incorporating culture, values, rituals, myths, customs, code of conduct, folklore (see Figures 2, 3 and 5) ethno-botanical knowledge and traditions etc that existed before Vedic civilization (1700-1100 B.C.) in South Asia mainly transmitted orally from Kiranti ancestors to their new generations.

Before the invention of writing systems, the Mugdum was and is still passed on by word of mouth and folklore (see Figure 5) and even after the invention of writing systems its bigger chunk has remained unwritten because of its practitioners are very reluctant to do so for the sake of its effectiveness. As a result, the unrecorded oral knowledge has been disappearing with its practitioners for ages. Some of its written form or main genres in Kiranti-Yakthung Pan include-Soksok, Yehang and Sapji. Soksok mainly records the stories of creation of the universe, the beginning of mankind, the cause and effect of the sins, the creation of evil spirits, such as the evil spirits of Envy, Jealousy and Anger and the cause and effect of death in childhood.

The Yehang narrates the story of the first leader of mankind who made laws for the sake of improvement of human beings from the stage of animal life to the enlightened human life and ways to control them by giving philosophical knowledge and teachings on spiritualism. Sapji states some classes of spirits like-the Good and the Evil, Inspiring, Tantric Feat, Envy and Jealousy. As the Kirant(i) people in the beginning were rational idolaters, they neither had temples, altars nor images, conceiving that none of these was necessary, but worshiped spirits whom they believe to be the residents of fire, the sun (cf. Chemjong 18-26) and nature as a whole.

Several references of 'Kirata' (< Oirat -> Kirat $->$ Kirant $->$ Kiranti cf. Rapacha 2009b) in Hindu mythological texts prove that the Mugdum also as an ancient Kiranti cosmology existed before and around Babylonian cosmological (1900-1200 BC) worldview whereas the Hindu Rigveda, then dates back to $1700-1100$ BC only. Thus, the Kiranti Mugdum since time immemorial is Kiranti people's cosmological worldview besides being a plethora of source for indigenous knowledge in their survival strategy. This knowledge now on must be incorporated in our education curriculum.

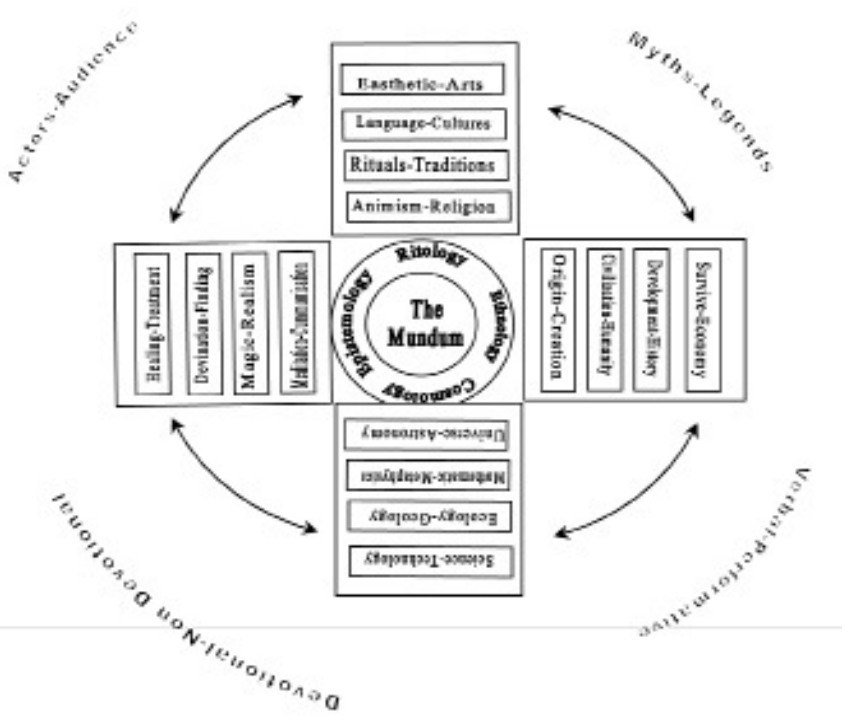

Figure 3: A distinct-indigenous Kiranti Mugdum and its interrelated sciences Source: Adapted from Rai 2013: 71 


\section{INCLUSIVE CURRICULUM}

Associated mainly with indigenous cultures and practices of life, there is a distinct world view between the nationally dominant or western scientific culture that the former believes in "survival for all" whereas the latter in "survival for the fittest" (Figure 4). For many Native educators, Stephens argues that culturally responsive science curriculum has to do with their (Alaskan IPs) passion for making cultural knowledge, language and values a prominent part of the schooling system (10). It has to do with presenting science within the whole of cultural knowledge (Figures 2, 3, 4, 5) in a way that embodies that culture (the Traditional Native Knowledge circle in Figure 4), and with demonstrating that science standards can be met in the process. It also has to do with finding or innovating the knowledge, strategies and support needed to carry out this work. For those educators not so linked to the local indigenous culture, culturally responsive science curriculum has more to do with connecting what is known about Western

Science education to what local people know and value (the

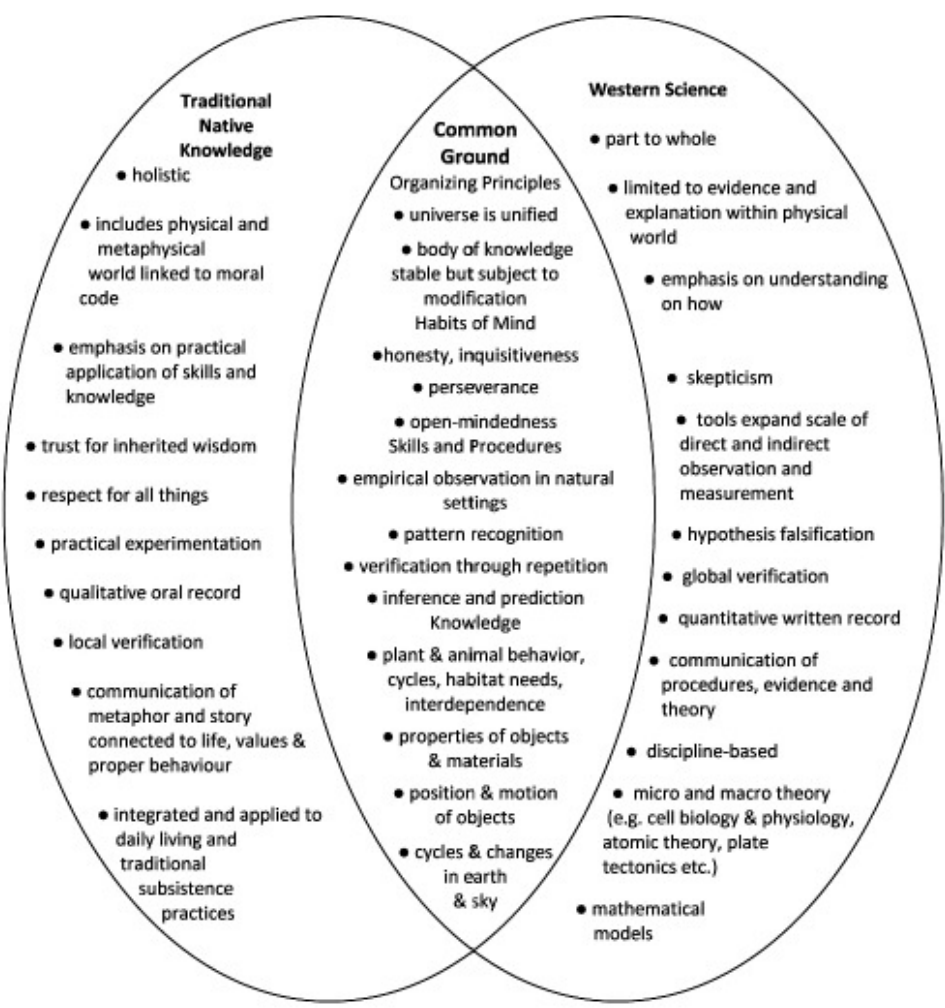

Figure 4: 'Survival for all vs. Survival for the fittest' associated with IK, TK and Western Science Source: Stephens 2000: 10 Western Science circle).

The implications for the learning processes imbedded in the three domains of knowledge represented in the overlapping circles (Figure 4) are numerous and of considerable significance. Stephens (10-11) highlights some of the implications for how we approach education hence-although educators obviously differ in their perspective, there is no doubt that the creation of culturally responsive science (or social sciences) curriculum has powerful implications for students for at least three reasons. The first is that a student might conceivably develop all of the common ground skills and understandings while working from and enhancing an IK or TK base. The second is that acquisition of the common ground, regardless of route, is a significant accomplishment. And the third is that exploration of a topic through multiple knowledge systems can only enrich perspective and create thoughtful dialog amongst local, national and international communities, which our elite Nepalese academia should without delay understand and take immediate action by making our curriculum inclusive. 


\section{Crossing the Border: International Journal of Interdisciplinary Studies}

\section{INVENTORY OF AESTHETICS AND FOLKLORE}

Besides survival knowledge and skills directly related to ethnoeconomics, indigenous peoples' aesthetics and folkloric knowledge (Figure 5) is also very rich accustomed to their local ethnocosmology. As an example of our inventory here we elicit an example of from the Kiranti-Yakthung people of Pallo Kirant area of east Nepal to campare some possible knowledge of Kiranti-Kõits folklore-as a component of Mugdum ( Mundhum Kiranti-Yakthung variety also cf. Subba incorporates-proverbs, folksongs like Palams and Khyali, cultural songs like Hakpare, riddles, various folk sayings, puns, charades, folktales and folk narratives in Kiranti-Yakthung (cf. Limbu, Figure 5) and in the rest Kiranti linguistic communities from historically homogenous homelands of eastern part of the country. Figure 5 shows that it is a superordinate term which incorporates various entities such as legends, myths, folklores, prehistoric accounts, sermons and moral, philosophical and healing exhortations both used in poetic rhythmic trance and in aesthetic discourse.

\section{Figure 5: Kiranti-Yakthung Folkloric knowledge}

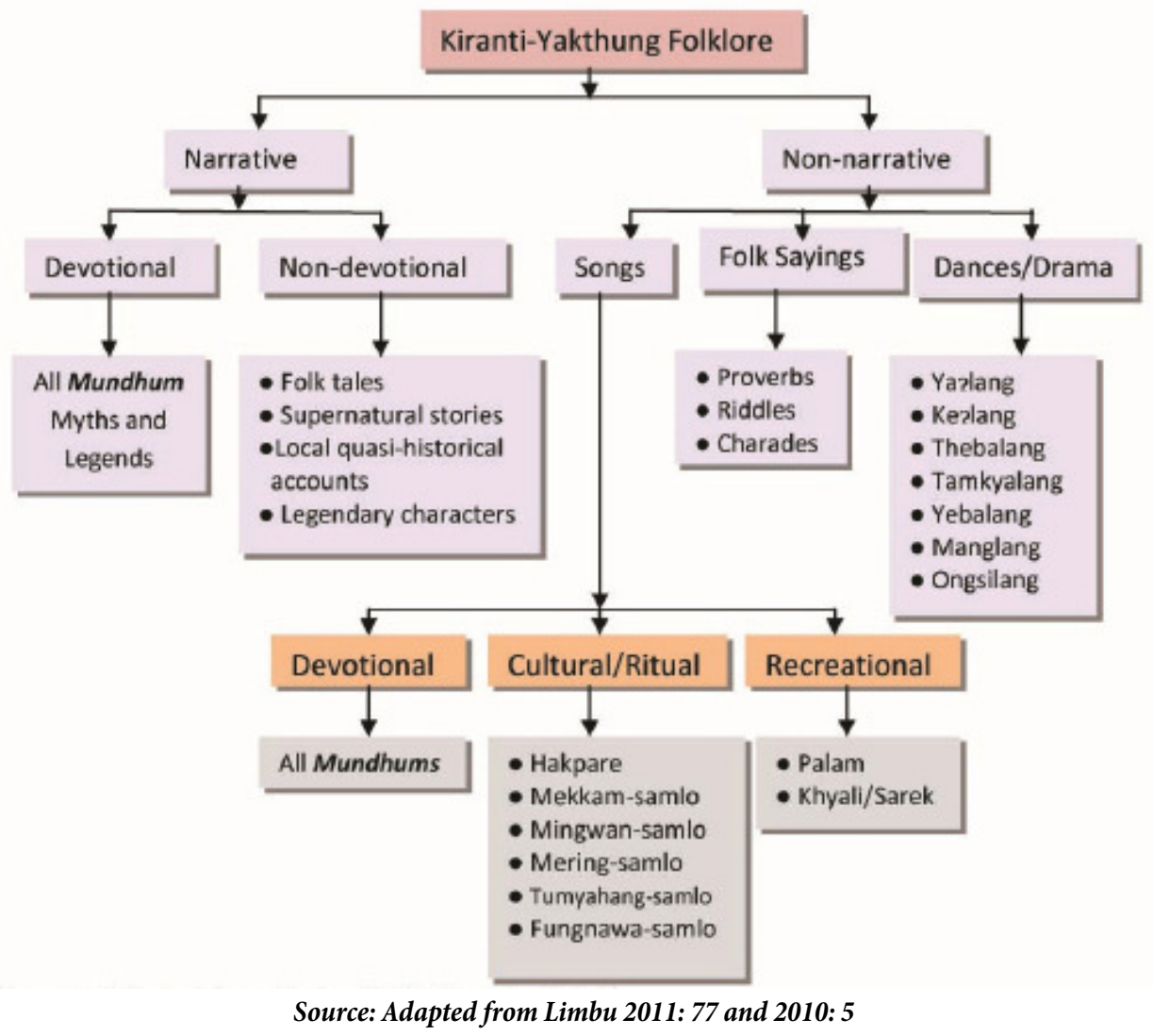

Traditionally, the Kiranti-Yakthung youths sing Palam in their daily life on the way to forests, in the working fields and perform Yarlang 'paddy dance' (Figure 5) on various socio-cultural occasions of various rituals like wedding ceremony, death rites, fair and in festivals as Kiranti-Kõits people dance Shyãdar 'cultural dance' (Figure 2 branched to ritualogy-CFF). Elderly Yakthungs, as do Kiranti-Kõits elders, narrate 


\section{INCLUSIVE CURRICULUM}

various types of secular and supernatural stories, and many folktales and additionally narrated by Phedanngma, Samba (not of Brazilian origin but a chance resemblance only), Yeba, Yema, Mangba, Yuma and Ongsi 'socio-cultural agents' identified as Põibo, Gyami and Nhaso in Kiranti-Kõits. All these kinds of Kiranti folk literature and aesthetics (Figure 6) are integral parts to Kiranti-Yakthung folklore as in Kiranti-Kõits and in the rest of Kiranti linguistic communities. The Kiranti-Yakthung Mundhum consisting of oral traditions, myths, songs, dances, legends, stories, tales, riddles, charades, drama, arts and above all the Yakthung Mundhum performance. Any indigenous peoples' narrative folklore can be one of the main sources of indigenous knowledge in dissecting the same source of knowledge in different taxonomy of ethnobotany and ethnomedicine (cf. Rapacha 21-22) according to one's socio-cultural necessity as it is clear in Kiranti-Kõits aesthetics (Figure 6).

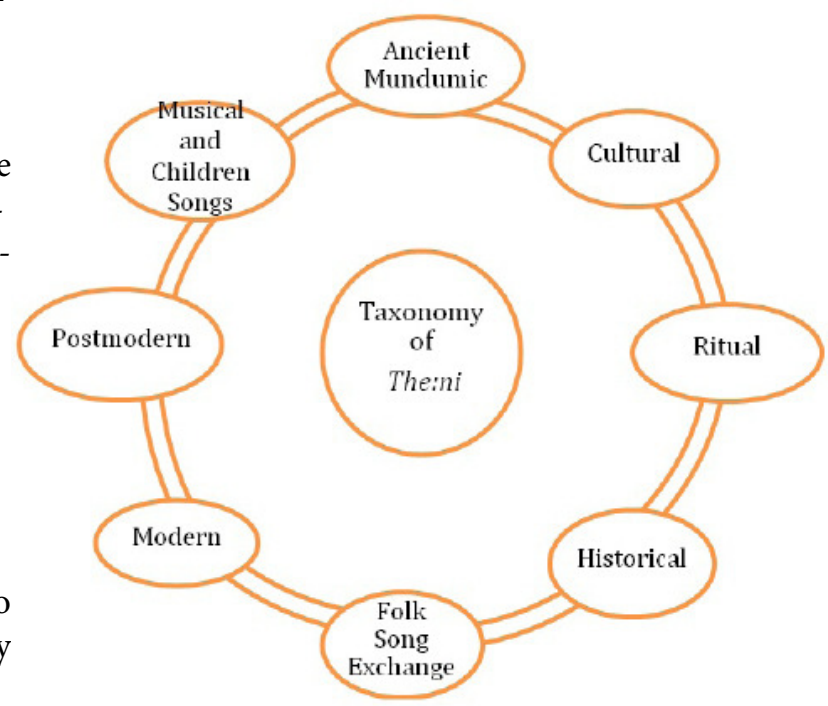

Figure 6: Kiranti-Kõits aesthetic knowledge Source: Translated from Rapacha 2013: 12

Their indigenous aesthetics known as The:ni (Mukhiya 12) has unique taxonomic characteristics, aesthetic value and identity as shown in Figure 6. Its uniqueness lies in sense, taste and creativity of the indigenous Kiranti-Kõits folks-daughters and sons of the soil of their indigenous territory. This can also be another sublime area of academic as well as vocational teaching and research yet unknown and unfamiliar to our native scholars in our universities by making our curriculum inclusive one.

\section{RECENT DEVELOPMENTS AT HOME AND BEYOND}

In connection to these inventories of some selective and limited (unlimited yet to be discovered) bits and pieces of new knowledge here, our oldest academic institution of higher studies-Tribhuvan University (TU) in the country very recently has decided to open up a new Department of Folklore Studies including seven other subjects like International Relations and Diplomacy, Social Work, Tourism and Hospitality, Sports Science, Dance, Fashion Design and Development Studies ${ }^{8}$. After a half century's slumber, TU's elite as well as power-slave officials and professors' awakening towards such students' need-based subject and curriculum may draw their attention and halt rat-races towards foreign universities, however it is not going to make any difference for the ages-old marginalized indigenous academia, peoples and students due to cronyism, discriminatory and exclusionary policy. During the course of Course of Study (in the sense of curriculum) preparation for the aforesaid Department of Folklore Studies, one of its committee members Prof Dr Jayaraj Pant had met me for consultation-and my indication to him was towards inclusion- 


\section{Crossing the Border: International Journal of Interdisciplinary Studies}

"indigenous academia including students and peoples now do not want to remain mere passive clapper-audiences but they want to be active players or main actors". Since then he disappeared and only once met on the phone-call perhaps being unable to digest my advice what I wanted to contribute in the process of making folklore studies department and its content genuinely inclusive. And without further information and consultation-forget about Free and Prior Informed Consent-my eyes fell on the newspaper line (Footnote 8's) about the department's establishment which made me skeptical that the newly opening Department of Folklore Studies very possibly will not be very different from that of Department of Linguistics infested with exclusionary-xenophobia of teachers from indigenous groups.

Recently, Dr Bishnu Pariyar in his BBC interview ${ }^{10}$ revealed that the University of Leeds is running a course on 'Natural Resource Management in Nepal' whereas our universities including TU in Nepal do not pay any attention to the plethora of knowledge/skills and resources (including human resource) presented in our inventories in previous sections and paragraphs. It suggests that our educational institutions along with national education system very sooner or later must opt for paradigm shift in its thought and action too.

\section{CONCLUSION}

This article with some selective and limited (unlimited yet to be explored) empirical inventories of indigenous knowledge system has tried to argue for the unavoidable need of inclusive curriculum in Nepal's technical and higher education policy and planning which will hopefully have positive impact in national education content and system. Also, inclusive curriculum in education best works as 'affirmative action' for peaceful socio-economic transformation of Nepal in recent developmental jargon. When observed seriously and comprehensively, Nepal's possibilities in her (its) tangible and intangible cultural and knowledge heritage including languages, cultures, archaeology, belief systems, ethnobotany, ethnomedicine etc) and skills are immense and abundance. Inclusiveness or participatory approach in our national curriculum, in fact can assure new paradigm shifts, e.g. from 'survival for the fittest' to 'survival for all' (an in Figure 4) since the reasons behind inclusive curriculum is obviously much more advantageous academically and socio-economically as discussed in this paper. If one argues for the impossibility of this new paradigm shift in any pretentions in our proposed modality of inclusive education policy, programme, content and system, then establishment of Nepalese University of Indigenous Studies (NUIS for short) will be a dire need of the hour to make happen the need-based new paradigm shift possible.

\section{ENDNOTES}

1. See UNESCO's Convention for the Safeguarding of the Intangible Cultural Heritage (2003) which Nepal has ratified in 2010.

2. http://www.latrobe.edu.au/nclusive curriculum accessed on Saturday, 31 Aug. 2013.

3. cf. Rapacha 2005, 2009a and elsewhere for a detailed identity crisis debates regarding the exonyms Sunuwar, Sunwar, Bhujuwar, Pirthwar and so on.

4. Some scholars argue that this is only a linguistic family but not indigenous group.

5. See 'Yarsa worth millions flown to Kathmandu', The Himalayan Times, 17 Sep. 2013. 


\section{INCLUSIVE CURRICULUM}

6. See 'Export Earnings from Medicinal Herb Soar', The Kathmandu Post, XXI \# 30, 19 Mar. 2013.

7. See 'Handicraft Exporters Demand Simplified Export Documentation', The Kathmandu Post, XXI \# 218, 24 Sep. 2013.

8. See “Tribima Nepali Lokjivan," Kantipur daily, 21 \# 222, 11 Asoj 2070 [22 Sep. 2013].

9. Personal communication with Prof Dr Pant, who had visited my office on Sunday, 7 July 2013 (date and event record in my personal Daily Diary-2013).

10. The BBC interview with Dr Bishnu Pariyar was aired on Wednesday, 2 September 2013. Dr Pariyar, Environmental Social Scientist and Policy Analyst, is affiliated as a Postdoctoral Research Fellow at School of Geography, University of Leeds, UK. He has earned his Doctor of Philosophy (Ph.D. 2006- 2011) in Social Policy and Environment from the University of York.

\section{WORKS CITED}

Bayly, Susan. Asian Voices in a Postcolonial Age: Vietnam, India and Beyond. New Dehli: Cambridge University Press, 2007.

Bhattarai, Anand M. "Protection of Himalayan Biodiversity: Exploring a Regional Legal Regime". Paper presented at Humboldt Colloquium as Humboldt Lecture Series, CEDA, Tribhuvan University, Kirtipur organized by Kathmandu Humboldt Club, 16 Sep. 2011.

Chemjong, Iman S. History and Culture of the Kirat People (Part I-II). Lalitpur: Kirat Yakthung Chumlung, 2003 [orig. 1967].

Chemjong, Iman S. and Nembang, Til Bikram (alias Bairagi Kaĩla). Limbu-NepaliAngreji Shabdakosh [Limbu-Nepali-English Dictionary]. Kathmandu: Royal Nepal Academy, VS 2059.

Fournier, Alain. "A Preliminary Report on the Puimbo and the Ngiami: The Sunwar Shamans of Sabra". Spirit Possession in the Nepal Himalayas. Ed. John T. Hitchcock and Rex L. Jones. Warminster: Aris \& Phillips, 1976 [orig. 1971]. 100-123.

Höfer, András. The Caste Hierarchy and the State in Nepal: A Study of the Muluki Ain of 1854. Lalitpur: Himal Books, 2004 [orig. 1979].

Koirala, Rishi R. "Country Monographs on Traditional System of Medicine." Unpublished report, Ayurveda Health Home P. Ltd., 2007.

Kunwar, R. and Bussmann, R.W. "Ethnobotany in the Nepal Himalaya: a review". Journal of Ethnobotany and Ethnomedicine, 4: 24 (2008): doi:10.1186/1746-42694-24.

Lama, Yeshi Choden, Ghimire, Suresh K and Aumeeruddy-Thomas, Y. Medicinal Plants of Dolpo: Amchis' Knowledge and Conservation. Kathmandu: WWF Nepal, 2001.

Leuthold, Steven. Indigenous Aesthetics: Native Art, Media and Identity. Texas: University of Texas Press, 1998.

Limbu, Ramesh K. "Ya?lang: An intangible Limbu Cultural Heritage". Contributions to Nepalese Studies, 38.1 (2011): 75-92.

---. "Performance in Limbu Mundhum: A Study of Cultural Representation". Diss., Tribhuvan University, Kirtipur, 2010.

Mukhiya, Atit. Adivasi Saundaryasastra Kiranti-Kõits (Sunuwar) The:niko Ek Saidhantik Addhyan [Indigenous Aesthetics: A Theoretical Study of Kiranti-Kõits 


\section{Crossing the Border: International Journal of Interdisciplinary Studies}

(Sunuwar) The:ni]. Kathmandu: Research Institute for Kirãtology, 2013.

Parker, Anne Z. Multi-Ethnic Interface in Eastern Nepal: Culture Change in Siddha. Pokhari. Lalitpur: Himal Books, 2013.

Peters, Larry. Tamang Shamans: An Ethnopsychiatric Study of Ecstasy and Healing in Nepal. India: Nirala Publications, 2007.

Pradhan, Kumar. The Gorkha Conquests: The Process and Consequences of the Unification of Nepal, with Particular Reference to Eastern Nepal (2nd Edition). Lalitpur: Himal Books and Social Baha, 2009 [orig. 1991].

Purcell, T.W. "IK and Applied Anthropology: Questions of Definition and Direction". Human Organization 57.3 (1998): 258-72.

Rai, Parshuram (alias Praveen Puma). "Performative Elements in Parusewa Mundum of Puma Community: A Study of Ritual Representation". Diss., Tribhuvan University, Kirtipur, 2013.

Rapacha, Lal-Shyãkarelu. 'Simanta Saundarya Kõits The:ni' [Marginalized aesthetics Kõits The:ni], Dzatnamuili 8.8 (2013 [VS 2070]): 10-13.

---. "IK Systems in Kiranti-Kõits Mugdum." Readings on Indigenous Culture and Knowledge [Culture and Development in Nepal Series-II], II (2011): 13-32.

---. Contemporary Essays on Vanishing Ethnicity, Cultures and Languages of Nepal: A Focus on Kiranti-Kõits. Kathmandu: Research Institute for Kirãtology, 2009a.

---. “A Descriptive Grammar of Kiranti-Kõits.” Diss., Jawaharlal Nehru University, New Delhi, 2005.

Rapacha, Lal and Jailab Thulung Rai. "A Study on Indentifying and Promoting Indigenous Peoples' Skills in Nepal". Unpublished report submitted to Employment Fund Secretariat (FES), HELVETAS, Lalitpur, 2013.

Saul, Rebecca. "Indigenous forest knowledge: Factors Influencing Its Social Distribution." Anthropology of Nepal: Peoples, Problems and Processes. Ed. Michael Allen. Kathmandu: Mandala Publications, 2005 1994]. 136-146.

Shrestha, Tirtha. "Rationale Behind Documentation of NTFPs and MAPs". Paper presented at Humboldt Colloquium as Humboldt Lecture Series, CEDA, Tribhuvan University, Kirtipur organized by Kathmandu Humboldt Club, 16 Sep. 2011.

Stephens, Sidney. Handbook for Culturally Responsive Science Curriculum. Fairbanks: Alaska Native Knowledge Network, 2000.

Subba, Chaitanya. "Nature and Indigenous Spirituality: The Context of Nepal." Journal of Nationalities of Nepal 2.2.4 (2000 [VS 2057]): 97-115.

WIPO. Intellectual Property and Traditional Knowledge, Booklet 2. New York: WIPO, 2005. 